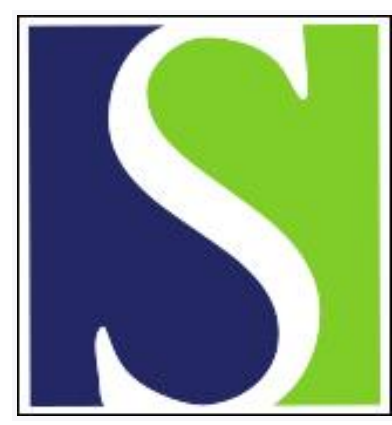

Scand J Work Environ Health 1979;5(3):167-177

https://doi.org/10.5271/sjweh.3090

Issue date: Sep 1979

\title{
Circadian rhythms in human performance
}

by Rutenfranz J, Colquhoun WP

Affiliation: Institut für Arbeitsphysio!ogie an der Universitlit Dortmund, Ardeystrasse 67, D-4600 Dortmund 1, Federal Republic of Germany.

Key terms: circadian rhythm; human performance; individual difference; situational factor; task factor

This article in PubMed: www.ncbi.nlm.nih.gov/pubmed/20120564

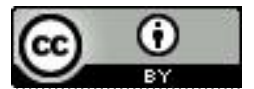




\title{
Circadian rhythms in human performance ${ }^{1}$
}

\author{
by JOSEPH RUTENFRANZ, M.D., Ph.D., ${ }^{2}$ and W. PETER COLQUHOUN, Ph.D. ${ }^{3}$
}

\begin{abstract}
RUTENFRANZ, J. and COLQUHOUN, W. P. Circadian rhythms in human performance. Scand. $j$. work environ. \& health 5 (1979) 167-177. Circadian rhythms in performance exist for a wide range of tasks studied under laboratory conditions; they also occur in measures of efficiency obtained in real-life situations. The rhythms appear to be related to the daily cycle of sleep "need," and the largest negative variations in performance are normally observed when this need is greatest. The detailed forms of the circadian functions are influenced to some extent by individual differences in age and personality. However, task factors are of equal, if not greater, importance in determining the nature of a performance rhythm, since its basic phase is markedly different in cases involving memory. This latter finding raises the practical problem of job design in relation to time of day, and a taxonomic approach is suggested as the most fruitful way of arriving at the optimal solution for particular work situations requiring different abilities.
\end{abstract}

Key words: circadian rhythms, human performance, individual differences, situational factors, task factors.

Daily variations in human performance efficiency have long been recognized. As early as 1893 von Bechterew (3) reported on "changes in speed of the psychic processes at different times of day" observed in investigations by his students Ostankow and Gran. He summarized their results as follows:

The speed of the psychic processes shows regular typical variations in the course of the day, and the type of daily variation remains more or less constant

1 Dedicated to Prof. T. Hellbrügge on his 60 th birthday.

2 Institute of Work Physiology at the University of Dortmund, Federal Republic of Germany.

3 MRC Perceptual and Cognitive Performance Unit, University of Sussex, Brighton, England.

Reprint requests to: Prof. J. Rutenfranz, Institut für Arbeitsphysiologie an der Universität Dortmund, Ardeystrasse 67, D-4600 Dortmund 1, Federal Republic of Germany. under normal conditions. This constancy reminds us of the course of physiological processes (for example body temperature). The type of daily variation is represented in the form of one and the same curve. The course of the psychic processes is retarded in the morning and accelerated in the evening. The lowest speed occurred in the afternoon [p. 291].

In the very same year Kraepelin (27) published similar observations and concretely described the daily course of the "psychic disposition" with the following words:

With continued work the performance usually rises until noon. Soon after the meal it has decreased very significantly. Simultaneously, a predominance of external associations and lower stability of imaginative combinations can be shown. These phenomena are no indication of work fatigue, since they disappear after $2-3$ hours, even when work is continued. [After the noon meal] the performance again increases slowly. Sooner or later however, without fail, fatigue gains the upper hand [p. 593]. 


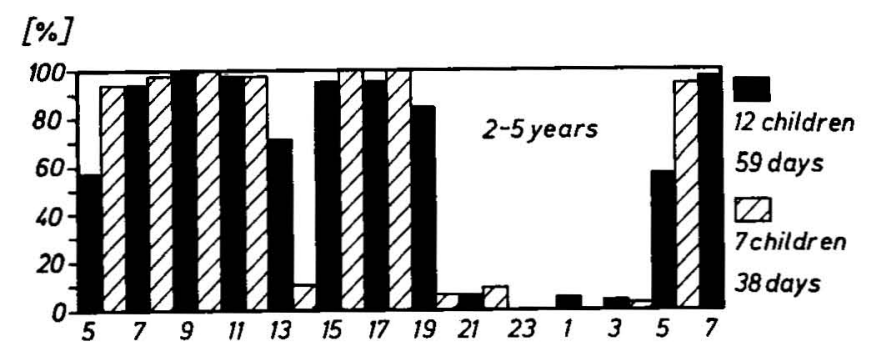

Fig. 1. Percentage of children awake at different times of the day in the following three age groups: $2-5$ years, $6-10$ years, $11-15$ years (21).
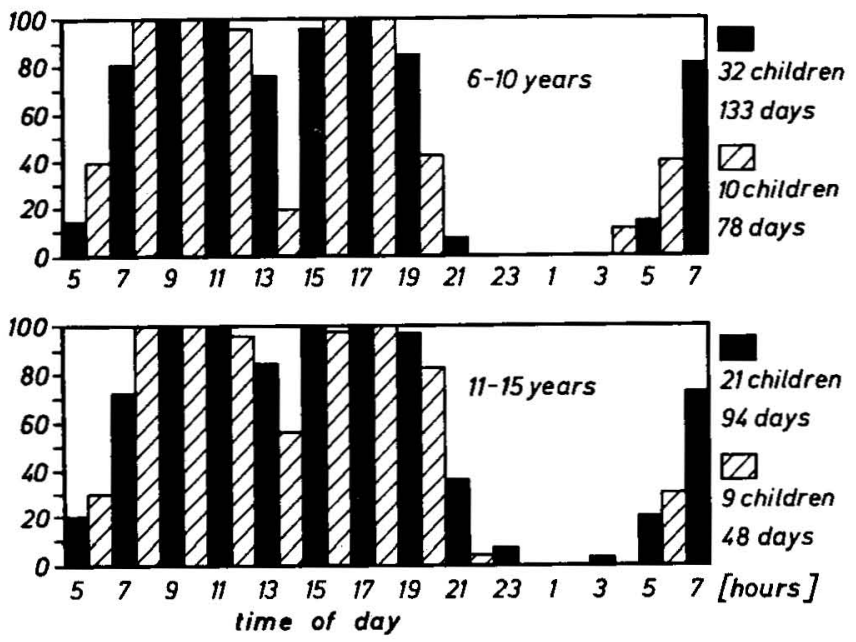

Characteristic of the reports of both authors is that they describe diurnal variations in human performance and the fact that these variations do not solely reflect the effects of work fatigue and meals. Although von Bechterew and Kraepelin only observed variations in performance during the day, much greater overall variation can be expected when the night hours are included, since this is the time when sleep normally occurs and sleep itself can be considered as the zero level of performance. It follows that the largest negative variations of performance are generally likely to be observed at those times when sleep can spontaneously occur during the course of the 24-h day.

As an example of the spontaneous occurrence of sleep, we would like to draw attention to an investigation of children (21). Children were allowed to sleep whenever they felt tired, and their sleeping/ waking patterns were observed. It was shown very clearly that, in the case of the youngest children (aged 2-5 years), 50\% were already awake at $0500,80 \%$ were asleep at $1400,90 \%$ were again awake at 1500 , and 80 to $90 \%$ had fallen asleep once more by 2000 . On the other hand, in the age group 11-15 years, more than $50 \%$ of the children did not awaken in the morning until 0700 , at 1400 only $30-$ $40 \%$ of them were asleep, at 1500 all the children were again awake, and not until 2200 had about $90 \%$ of the children fallen asleep once more (fig. 1).

That these spontaneous variations of "sleep need" also exist in adult life is generally accepted. Their intimate connection with performance variation is evident from investigations of phenomena such as "falling asleep over the steering wheel." Prokop and Prokop (31) asked about 500 automobile drivers if they had ever momentarily fallen asleep over the steering wheel and, if so, when. The frequencies of positive answers in relation to time of day are shown in fig. 2. The only times when no positive answers were given were for 0700,0800 , and 1800 . Twenty-five percent of the cases occurred between 1200 and 1500 , and $58 \%$ of the ca- 
ses between 2300 and 0500 . The highest frequencies of positive answers were given for 1400 and 0000 .

It is apparent from investigations such as this that there are evidently times of day at which performance is relatively high. These times are interrupted by periods of increasing sleep need, in which performance gradually diminishes or is reduced to zero. Sleep and performance can thus be seen as antagonistic phenomena.

\section{INDUSTRIAL PERFORMANCE RHYTHMS}

If circadian rhythms exist in human performance, then such rhythms might be expected to be reflected in daily industrial production figures at different times, since, as Vernon (34) supposed, "experienced industrial workers unconsciously adopt habits of work which tend to the production of a maximum output with a minimum of effort." Contrary to this supposition, however, Vernon could not find a uniform rhythm in the production curves of various munitions workers. Many other subsequent investigations have reached similar conclusions.

From the few studies in which industrial performance rhythms have been reported, we would like to give one example of a change in the "accuracy" aspect and one of change in the "speed" aspect of performance. The best-known example of circadian variation in accuracy is the investigation of Bjerner et al. (4). These authors analyzed 75,000 mistakes made in the reading of gas meters and found (fig. 3 ) that two clear peaks occurred - one at 0300 and another at 1500 . The fewest mistakes were made between 0700 and 0900 although the error rate was also low between 1700 and 1900 .
Fig. 2. Percentage of 569 automobile drivers admitting to "falling asleep over the steering wheel" at different times of the day (31).
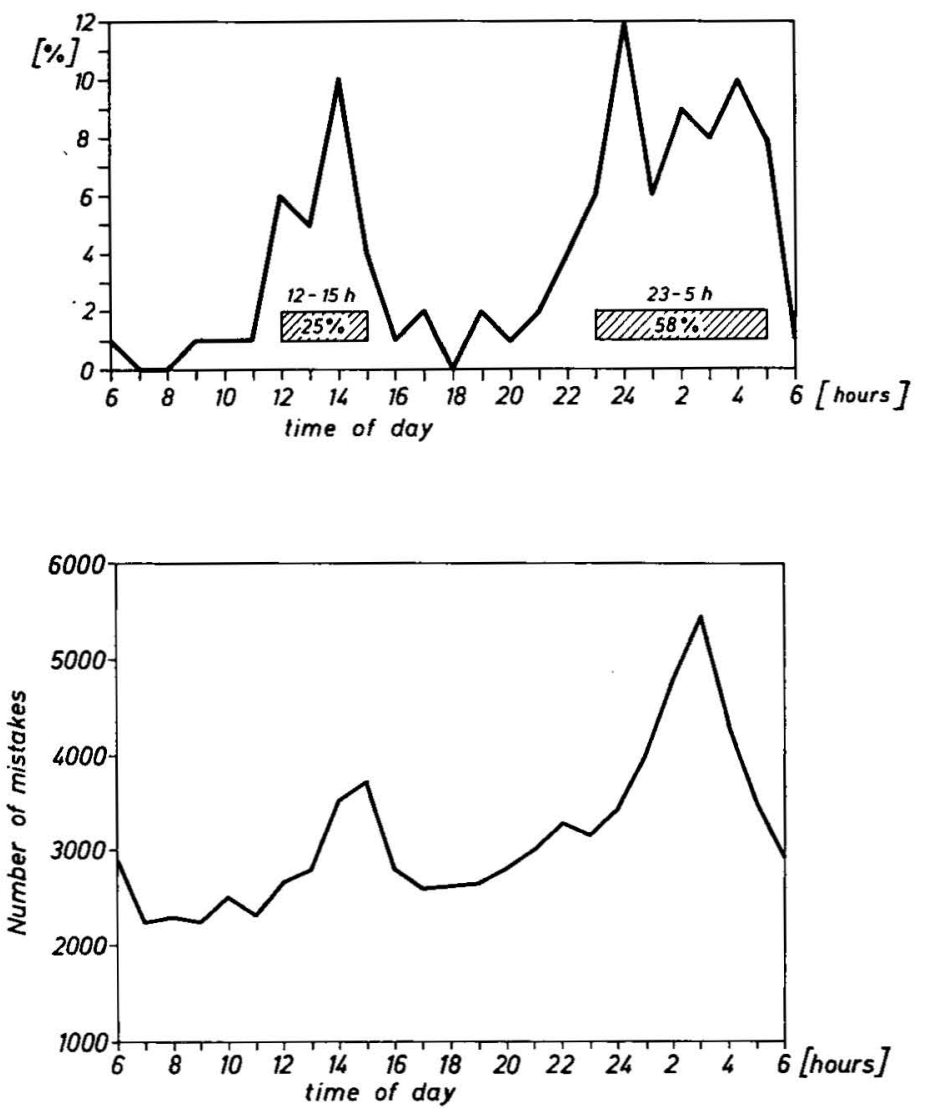

Fig. 3. Number of mistakes made by meter readers in a gas works, as determined from checks on their records (4). 
As regards the speed aspect, Goldmarck and Hopkins (19) showed that work speed in two large American firms rose continuously in the morning until 1100 , slowed down at noon, then increased again in the afternoon until a point in the late afternoon when it slowed down once more.

Graf (20) later pointed out that circadian rhythms in industrial performance may sometimes be difficult to detect because the workers attempt to mask such variations in productivity out of fear of changes in their piecework wages. If however there exists a trust relationship with a group of workers who are given definite amounts of work for equal periods of time and are encouraged to take a break after the completion of each of these amounts of work, then clear variations in working speed do come to light. It seems likely, therefore, that industrial performance rhythms would be seen with far greater frequency were it not for the obscuring effects of motivational and situational factors.

\section{LABORATORY PERFORMANCE RHYTHMS}

Diurnal variations in human performance have been studied under laboratory conditions with increasing frequency over the last few decades. An extensive summary of the early literature is given in the review by Freeman and Hovland (17). Excluding night experiments, they found "four different kinds of relation between performance and time of day: (1) a continuous rise through the day, (2) a continuous fall, (3) a morning rise and an afternoon fall, and (4) a morning fall and an afternoon rise." As Hockey and Colquhoun (22) say,

The reasons for this are most probably to be found in poor methodology and in inadequate or inappropriate techniques of performance measurement, though there is an additional possibility that different kinds of task give rise to different diurnal trends [p. 3].

The experiments reviewed by Freeman and Hovland (17) were conducted mainly with only a few subjects or with groups of subjects for just one day. In addition, practice effects were not controlled in most of the studies. It is now universally recognized that the following methodological prerequisites are imperative:

(a) adequate pretraining and subsequent control for practice effects where necessary (e.g., with the use of special experimental designs such as Latin squares);

(b) reduction of individual influences either with the use of groups of substantial size in "single cycle" studies or with the observation of behavior over several successive cycles (preferably at least 10).

The largest recent study which takes these conditions into consideration is that by Blake (5) in which, with the use of the "single cycle" paradigm, sets of 25 or 30 subjects were tested five times during the day over the period from 0800 to 2100 . Hockey and Colquhoun (22) summarized the procedure as follows: Each set performed a different task; these tasks were: (a) self-paced five-choice serial reaction [this can be considered as essentially a "motor" version of the color-naming task used by Kleitman (25)], (b) vigilance detection of infrequent "long" tones in a regular sequence of tones of standard duration, (c) card sorting, (d) letter cancellation, (e) time estimation (production method), (f) short-term memory (digit span), (g) simple reaction time to a visual stimulus, and (h) calculation (addition of columns of two-digit numbers).

Fig. 4 shows the results for those test scores for which a statistically significant difference was found between performance levels at different times of day. Although not all of the scores show exactly the same "over-day" trend, in the majority of cases performance increases sharply between 0800 and 1030, and a "postlunch dip" is almost universal. However it is also true that in all except one case the maximum level is seen at 2100 , whether or not there is a clearly discernable earlier performance peak in the morning.

Artifacts possibly contributing to this "late peaking" phenomenon include a final surge of motivation ("end of workday" effect) or (conceivably) an interaction between the practice effect and time of day. (The Latin square design employed as- 
sumes this interaction to be zero.) However, it could be argued that the fact that performance of the digit-span task did not show this "late peak" implies that at least the first of these artifacts was not the cause. It seems probable, therefore, that the phenomenon is a genuine one.

The results suggest that there are two types of tasks: one (represented by six of the tests in Blake's series, all of which are essentially "immediate informationprocessing" tasks) exhibiting a rhythm that (perhaps coincidentally) is roughly in phase with the rhythm of body temperature and another (represented in this series only by the digit-span test - which involves storage of information) showing a rhythm that is out of phase with this function.

This phenomenon should not be a surprise, since it is known that different physiological processes themselves show different phasing in their circadian rhythms
(9) and a similar divergence might thus reasonably be expected to be seen in different psychological functions also.

Age, and its accompanying differences in sleeping time, is another variable which may affect the form of the diurnal performance curve. If we consider calculations, for example, Blake (5) used adult subjects for his experiment, whereas other workers have used children in their investigations of time of day effects on this task. Thus Rutenfranz and Hellbrügge (32) studied the performance of 11-year-old boarding-school children who were required to add columns of four one-digit numbers, but were not allowed to write down intermediate totals. The same authors also studied children in a school of "higher education"; in this case the subjects had to multiply the first two numbers and to add this product to the sum of the last two numbers. Seventeen children were tested over a period of $117 \mathrm{~d}$. The results are
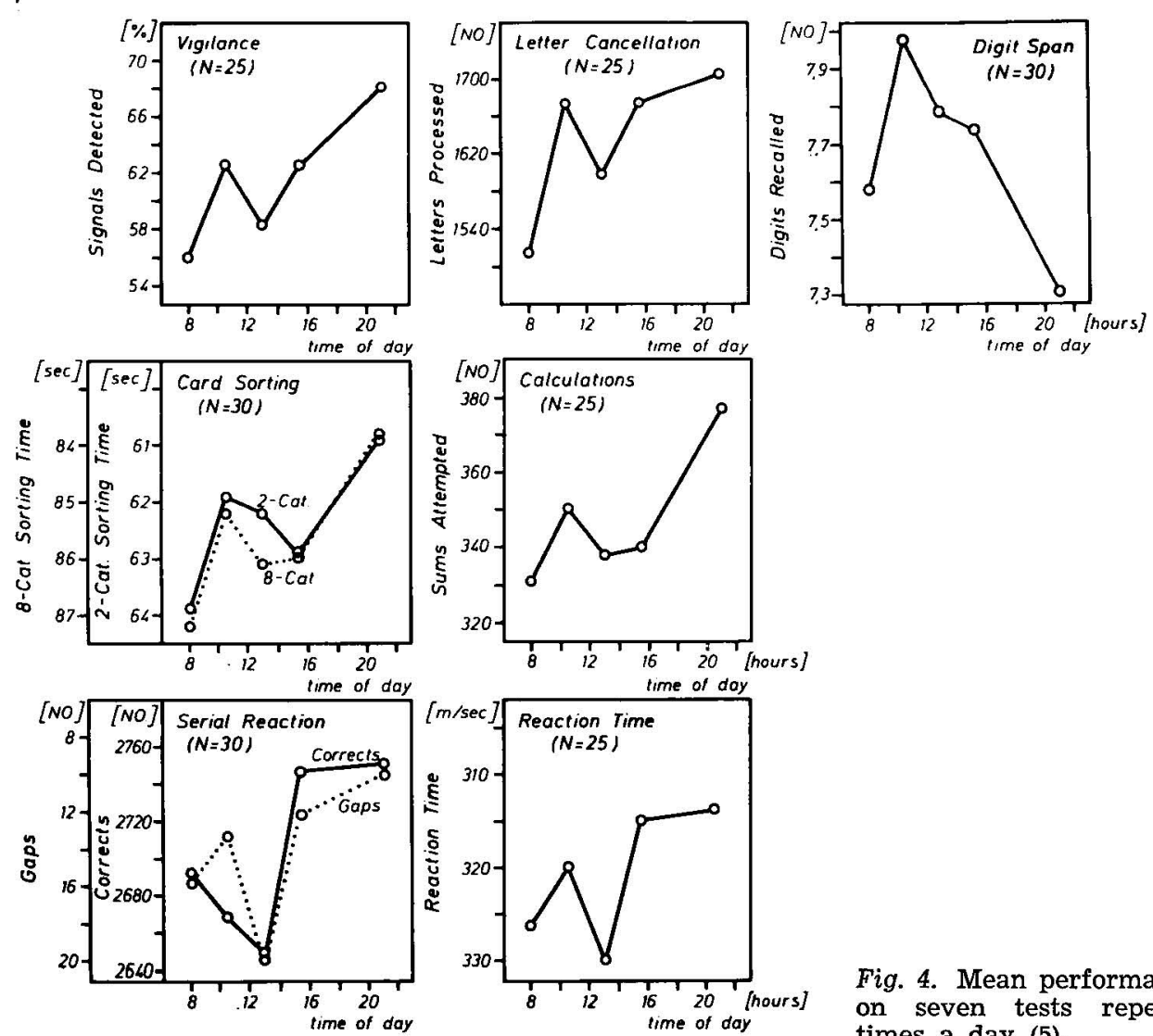

Fig. 4. Mean performance scores on seven tests repeated five times a day (5). 